\title{
A Novel Attractive Algorithm for Handling Systems of Fractional Partial Differential Equations
}

\author{
MOHAMMAD ALAROUD \\ Department of Mathematics \\ Faculty of Arts and Science \\ Amman Arab University \\ Amman, 11953, JORDAN
}

\author{
YOUSEF AL-QUDAH \\ Department of Mathematics \\ Faculty of Arts and Science \\ Amman Arab University \\ Amman, 11953, JORDAN
}

\begin{abstract}
The purpose of this work is to provide and analyzed the approximate analytical solutions for certain systems of fractional initial value problems (FIVPs) under the time-Caputo fractional derivatives by means of a novel attractive algorithm, called the Laplace residual power series (LRPS) algorithm. It combines the Laplace transform operator and the RPS algorithm. The proposed algorithm produces the fractional series solutions in the Laplace space based upon basically on the limit concept and then transforming bake them to original spaces to get a rapidly convergent series approximate solution. To validate the efficiency, accuracy, and applicability of the proposed algorithm, two illustrative examples are performed. Obtained solutions are simulated graphically and numerically. The analysis of results reached shows that the proposed algorithm is applicable, effective, and very fast in determining the solutions for many fractional problems arising in the various areas of applied mathematics
\end{abstract}

Key-Words: - Caputo-time fractional derivative; Fractional partial differential equations; Laplace transform residual power series algorithm; Fractional initial value problems.

Received: March 26, 2021. Revised: September 24, 2021. Accepted: October 12, 2021. October 20, 2021.

\section{Introduction}

During the past years, a lot of prominent contributions were made to the subject of the theory and applications of fractional partial differential equations (FPDEs). These equations are more effectively used to analyze and describe several phenomena in various fields such as mechanical systems, dynamical systems, control theory, mixed convection flows, heat transfer, unification of diffusion, image processing, and wave propagation phenomenon [1]-[7]. The nonlocal property is the most significant for using FPDEs in aforesaid applications and others. As a fact, that the differential operators and the integral operators of integer orders are local; however, the differential operators of fractional order and the integral operators of fractional order are considered nonlocal. In other words, the system's next state not only relies on its current state but also on its historical states. Actually, this is deemed the primary reason why the fractional order differential operators can present an outstanding instrument to describe the memory and hereditary properties of numerous mathematical processes. For more details about the FPDEs, together with their applications [8]-[12].
Several numerical methods are utilized to solve a lot of fractional problems in various areas. The most feature of the numerical method is that we can attain a numerical answer even if the given problem does not have an analytical solution. In certain situations, FPDEs are analytically solved, where it is limited to the linear one and hard to find their closed-form solutions to non-linear issues. Therefore, an effective, suitable analytical algorithm for the solutions of such equations is needed. Residual power series method, reproducing kernel method, homotopy analysis method, and differential transform method are some of advanced semianalytical methods that used to deal with different types of nonlinear PDEs [13]-[23]. The motivation of this article is to introduce the Laplace residual power series (LRPS) algorithm for solving systems of fractional initial value problems (FIVPs). The proposed method is suggested and proved by ElAjou [24] to investigate exact solitary solutions for a certain class of time-FPDEs. The LRPS solutions of the main problems can be obtained after converting and solving them in the Laplace space and then transform back the solutions into the main spaces. The method provides the components of the suggested fractional expansion via the concept of 
limit when the transform variable approaches infinity. Unlike, the RPS method that used fractional differentiation and hence, our method doesn't need several calculations and time in steps of obtaining the series solutions [25]-[27]. So, the unknown functions can be found by fewer time computations, and hence the approximate analytical solution can be constructed as a convergent multiple fractional series. For more real-world applications of fractional operators in physics and engineering, including quantum mechanics, heat flow, Schrodinger equations, control theory and so on, we refer to [28]-[33] and references therein.

The outline of this article as follows: In next section, some mathematical preliminaries are revisited. In Section 3, the procedure of finding the Laplace RPS series solutions is introduced. In Section 4, the efficiency and applicability of the proposed method are demonstrated via testing two suitable systems of FIVPs. Finally, a conclusion is made in the last section.

\section{Mathematical Preliminaries}

In this section, we recall the mathematical preliminaries of some fractional operators and Laplace transform operator. As well as, we review the essential theories and primary results related to fractional Taylor's formula in the Laplace space.

Definition 2.1 [1] For $\beta \in \mathbb{R}^{+}$, the RiemannLiouville fractional integral operator for a realvalued function $?(x, t)$ is denoted by $\mathcal{J}_{t}^{\beta}$ and defined as:

$\mathcal{J}_{t}^{\beta}$ 目 $(x, t)=$

$\left\{\begin{array}{ll}\frac{1}{\Gamma(\beta)} \int_{0}^{t} \frac{\square(x, \eta)}{(t-\eta)^{1-\beta}} d \eta, & 0<\eta<t, \beta>0 \\ 习(x, t) & \beta=0\end{array}\right.$.

Definition 2.2. [3] The time fractional derivative of order $\beta>0$, for the function $0(x, t)$ in the Caputo case is denoted by $\mathfrak{D}_{t}^{\beta}$, and defined as:

$\mathfrak{D}_{t}^{\beta}$ 圆 $(x, t)$

$= \begin{cases}\mathcal{J}_{t}^{n-\beta}\left(D_{x}^{n} \text { Q }(x, t)\right), & 0<n-1<\beta \leq n . \\ D_{x}^{n} \text { ? }(x, t), & \beta=n\end{cases}$

where $D_{x}^{n}=\frac{\partial^{n}}{\partial x^{n}}$, and $n \in \mathbb{N}$.

Definition 2.3. [24] The Laplace transform of the piecewise continuous function $](x, t)$ on $I \times[0, \infty)$ is defined as:

$$
\begin{aligned}
& \mathcal{L}\left\{D_{t}^{\beta} \text { 目 }(x, t)\right\}_{0}= \\
& \quad(x, s)=\int_{0}^{\infty} \vartheta(x, t) e^{-s t} d t, \quad s>\sigma,
\end{aligned}
$$

where $\sigma$ is the exponential order of $2(x, t)$.

Definition 2.4. [24] The Inverse Laplace transform of the function $[(x, s)$ is defined as:

$$
\begin{aligned}
& ?(x, t)=\mathcal{L}^{-1}\{\text { ? }(x, s)\} \\
& =\int_{a-i \infty}^{a+i \infty} \text { ? }(x, s) e^{s t} d s, \quad a=\operatorname{Re}(s)>b \text {, }
\end{aligned}
$$

where $2(x, s)$ is analytic transform function on the right half plane of the absolute convergence of the Laplace integral.

For $\mathcal{L}\{$ (⿴囗十 $(x, t)\}=$ ? $(x, s), \mathcal{L}\{\mathrm{Z}(x, t)\}=\mathrm{Z}(x, s)$, and $\lambda, \mu \in \mathbb{R}$. Following, some of the useful characteristics of the Laplace transform operator and its inverse operator which will be needed in this work as follows:
1) $\mathcal{L}\{\lambda \mathrm{Q}(x, t)+\mu \mathrm{z}(x, t)\}$

$$
=\lambda ?(x, s)+\mu \mathrm{Z}(x, s) .
$$
2) $\mathcal{L}^{-1}\{\lambda \mathrm{g}(x, s)+\mu \mathrm{Z}(x, s)\}$ $=\lambda \mathrm{?}(x, t)+\mu \mathrm{z}(x, t)$.
3) $\lim _{s \rightarrow \infty} s$ ? $(x, s)=?(x, 0)$.
4) $\mathcal{L}\left\{t^{m \beta}\right\}=\frac{\Gamma(m \beta+1)}{s^{m \beta+1}}, \beta>-1$.
5) $\quad \mathcal{L}\left\{\mathfrak{D}_{t}^{\beta}\right.$ 目 $\left.(x, t)\right\}=s^{\beta}$ ? $(x, s)-$ $\left.\sum_{j=0}^{n-1} s^{\beta-j-1}\right]_{t}(x, 0), n-1<\beta n, n \in \mathbb{N}$.
6) $\mathcal{L}\left\{\mathfrak{D}_{t}^{m \beta}\right.$ 目 $\left.(x, t)\right\}=s^{m \beta}$ 目 $(x, s)-$ $\sum_{j=0}^{m-1} S^{(m-j) \beta-1} \mathfrak{D}_{t}^{j \beta}$ 国 $(x, 0), 0<\beta \leq$ $1, m \in \mathbb{N}$.

However, El-Ajou [24] has been introduced and proved new results related to the generalized Taylor series formula in the Laplace space to identify the series solution of linear and non-linear FPDEs. Further, the requirements for convergence of the new series expansion is clarified and proved as follows:

Theorem 2.1 [24] Assume that the multiple fractional series of the transform function $0(x, s)=$ $\mathcal{L}\{[(x, t)\}$ has the following shape:

$$
\text { ? }(x, s)=\sum_{k=0}^{\infty} \frac{?_{k}(x)}{s^{k \beta+1}}, s>0, \beta \in(0,1] \text {. }
$$


then the unknown functions ${ }_{k}(x)$ will be in the form ${ }_{k}(x)=\mathfrak{D}_{t}^{k \beta}$ 团 $(x, 0)$, where $\mathfrak{D}_{t}^{k \beta}=\mathfrak{D}_{t}^{\beta} \cdot \mathfrak{D}_{t}^{\beta}$. $\cdot \cdot \mathfrak{D}_{t}^{\beta}$ (k-times).

Remark 2.1: The inverse Laplace transform of the series expansion in Theorem 2.1 has the following shape:

$$
\begin{gathered}
\text { ? }(x, t)=\sum_{k=0}^{\infty} \frac{\mathfrak{D}_{t}^{k \beta} \text { 国 }(x, 0)}{\Gamma(k \beta+1)} t^{k \beta}, t \geq 0, \beta \\
\in(0,1] .
\end{gathered}
$$

Theorem 2.3. [24] For $\beta \in(0,1]$. If

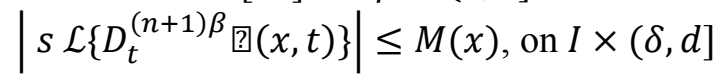
where $\mathcal{L}\{$ 回 $(x, t)\}=?(x, s)$, can be expanded as a multiple fractional series in Theorem 2.1, then the reminder $R_{m}(x, s)$ of the multiple fractional series (6) satisfies the following inequality:

$$
\left|R_{m}(x, s)\right| \leq \frac{M(x)}{s^{1+(m+1) \beta}}, \quad x \in I,
$$

\section{General Principle of the Laplace RPS Method}

In this section, we clarify the principle of the Laplace RPS algorithm to obtain the series solutions for fractional PDEs systems. Our strategy to use the proposed scheme depends on coupling the Laplace transform operator and fractional residual power series approach by means transferring the main system from the original space into the Laplace space, then solving the obtained Laplace system via employing the RPS approach, and as a last step, we transfer back the obtained Laplace series solutions to the original space to get on the multiple fractional PS approximate series solutions for the main system. More specifically, we consider the following initial value problems for a system of FPDEs:

$$
\left\{\begin{array}{l}
\mathfrak{D}^{\beta} u-F\left(u, v, D_{x}^{n} u, D_{x}^{n} v, D_{x}^{n} u v\right)=0 \\
\mathfrak{D}^{\beta} v-G\left(u, v, D_{x}^{n} u, D_{x}^{n} v, D_{x}^{n} u v\right)=0 . \\
u(x, 0)=\varphi(x), \quad v(x, 0)=\omega(x)
\end{array}\right.
$$

where $t \geq 0, x \in \mathbb{R}, \mathfrak{D}^{\beta}$ refers to $\beta$-th Caputo fractional derivative for $\beta \in(0,1], u(x, t), v(x, t)$ are two unknown functions to be determined. In this work, we assume that $u(x, t)$, and $v(x, t)$ satisfy the requirements for the existence of a unique solution and satisfies all conditions to have a multiple fractional PS representation at $t=0$.

To construct the approximate solutions of (8) by using the Laplace RPS approach, one can perform the following algorithm:
Step A: Apply the Laplace transform on both sides of (8), to get:

$$
\begin{aligned}
& U(x, s) \\
& =\frac{\varphi(x)}{s}+\mathcal{L}\left\{F\left(u, v, D_{x}^{n} u, D_{x}^{n} v, D_{x}^{n} u v\right)\right\} \\
& V(x, s) \\
& =\frac{\omega(x)}{s}+\mathcal{L}\left\{G\left(u, v, D_{x}^{n} u, D_{x}^{n} v, D_{x}^{n} u v\right)\right\}
\end{aligned}
$$

Step B: Assume that the approximate solutions of the Laplace system (9) take the following fractional expansions:

$$
\begin{aligned}
& U(x, s)=\frac{\varphi(x)}{s}+\sum_{m=1}^{\infty} \frac{u_{m}(x)}{s^{m \beta+1}} s>0, \\
& V(x, s)=\frac{\omega(x)}{s}+\sum_{m=1}^{\infty} \frac{v_{m}(x)}{s^{m \beta+1}} s>0 .
\end{aligned}
$$

and the $k$-th Laplace series solutions takes the following forms.

$$
\begin{aligned}
& U_{k}(x, s)=\frac{\varphi(x)}{s}+\sum_{m=1}^{\infty} \frac{u_{m}(x)}{s^{m \beta+1}} s>0, \\
& V_{k}(x, s)=\frac{\omega(x)}{s}+\sum_{m=1}^{\infty} \frac{v_{m}(x)}{s^{m \beta+1}} s>0 .
\end{aligned}
$$

Step C: We define the $k$-th Laplace fractional residual functions of (9) as:

$$
\begin{aligned}
& \mathcal{L}\left\{\operatorname{Res}_{k}(x, s)\right\} \\
& =U_{k}(x, s)-\frac{\varphi(x)}{s} \\
& -\mathcal{L}\left\{F\left(u, v, D_{x}^{n} u, D_{x}^{n} v, D_{x}^{n} u v\right)\right\}, \\
& \mathcal{L}\left\{\operatorname{Res}_{k}(x, s)\right\} \\
& =V_{k}(x, s)-\frac{\omega(x)}{s} \\
& -\mathcal{L}\left\{G\left(u, v, D_{x}^{n} u, D_{x}^{n} v, D_{x}^{n} u v\right)\right\},
\end{aligned}
$$

and the Laplace residual function of (9) are defined as:

$$
\begin{aligned}
& \lim _{k \rightarrow \infty} \mathcal{L}\left\{\operatorname{Res}_{k}(x, s)\right\}=\mathcal{L}\{\operatorname{Res} U(x, s)\} \\
& =U(x, s)-\frac{\varphi(x)}{s} \\
& -\mathcal{L}\left\{F\left(u, v, D_{x}^{n} u, D_{x}^{n} v, D_{x}^{n} u v\right)\right\}, \\
& \lim _{k \rightarrow \infty} \mathcal{L}\left\{\operatorname{Res} V_{k}(x, s)\right\}=\mathcal{L}\{\operatorname{ResV}(x, s)\} \\
& =V(x, s)-\frac{\omega(x)}{s} \\
& -\mathcal{L}\left\{G\left(u, v, D_{x}^{n} u, D_{x}^{n} v, D_{x}^{n} u v\right)\right\}
\end{aligned}
$$


As in [24], some useful facts of Laplace residual function which are essential in finding the approximate solutions are listed as follows:

- $\lim _{k \rightarrow \infty} \mathcal{L}\left\{\operatorname{Res} U_{k}(x, s)\right\}=\mathcal{L}\{\operatorname{Res} U(x, s)\}$, and $\lim _{k \rightarrow \infty} \mathcal{L}\left\{\operatorname{Res}_{k}(x, s)\right\}=\mathcal{L}\{\operatorname{Res} V(x, s)\}$,

for $x \in \mathbb{R}$, and $s>0$.

- $\mathcal{L}\{\operatorname{Res} U(x, s)\}=0$, and $\mathcal{L}\{\operatorname{Res} V(x, s)\}=$ 0 , for $x \in \mathbb{R}$, and $s>0$.

- $\lim _{s \rightarrow \infty} s^{1+k \beta} \mathcal{L}\left\{\operatorname{Res} U_{k}(x, s)\right\}=0$, and $\lim _{s \rightarrow \infty} s^{1+k \beta} \mathcal{L}\left\{\operatorname{ResV}_{k}(x, s)\right\}=0$, for $x \in \mathbb{R}, s>0$, and $k=1,2,3, \ldots$

Step D: Substitute the $k$-th Laplace series solution (11) into the $i$-th Laplace fractional residual functions of (12).

Step E: The unknown coefficients $u_{k}(x)$, and $v_{k}(x), k=1,2,3, \ldots$, could be founded by solving the systems

$$
\lim _{s \rightarrow \infty} s^{1+k \beta} \mathcal{L}\left\{\operatorname{Res}_{k}(x, s)\right\}=0,
$$

and $\lim _{s \rightarrow \infty} s^{1+k \beta} \mathcal{L}\left\{\operatorname{Res}_{k}(x, s)\right\}=0$. Then,

we collect the obtained coefficients in term of fractional expansions series (11)

$U_{k}(x, s)$, and $V_{k}(x, s)$.

Step F: Applying the inverse Laplace transform operator on both sides of the obtained series approximate solutions to get the multiple fractional PS approximate solutions $u_{k}(x, t)$, and $v_{k}(x, t)$ of the target system(8).

\section{General Principle of the Laplace RPS Method}

This section is devoted to illustrate the applicability and performance of the proposed method for solving two systems of FIVPs. All calculations have been carried out using the Mathematica software 12.

Example 4.1: consider the following linear system of FIVPs [36]:

$$
\left\{\begin{array}{c}
\mathfrak{D}_{t}^{\beta} u-D_{x} v+u+v=0 \\
\mathfrak{D}_{t}^{\beta} v-D_{x} u+u+v=0, \quad x \in R, t \geq 0, \\
u(x, 0)=\sinh x, \quad \beta \in(0,1] \\
v(x, 0)=\cosh x
\end{array}\right.
$$

For $\beta=1$, the exact solutions of (14) are $u(x, t)=$ $\sinh (x-t)$, and $v(x, t)=\cosh (x-t)$.
Firstly, By applying the Laplace transform operator on the system (14) and using the facts $\left\{\mathfrak{D}_{t}^{\beta} u(x, t)\right\}=s^{\beta} U(x, s)-s^{1-\beta} \sinh x \quad, \quad$ and $\left\{\mathfrak{D}_{t}^{\beta} v(x, t)\right\}=s^{\beta} V(x, s)-s^{1-\beta} \cosh x$, then we have the following Laplace system:

$$
\left\{\begin{array}{r}
U(x, s)=\frac{\sinh x}{s}+\frac{1}{s^{\beta}} D_{x} V(x, s) \\
-\frac{1}{s^{\beta}} U(x, s)-\frac{1}{s^{\beta}} V(x, s) \\
V(x, s)=\frac{\cosh x}{s}+\frac{1}{s^{\beta}} D_{x} U(x, s) \\
-\frac{1}{s^{\beta}} U(x, s)-\frac{1}{s^{\beta}} V(x, s)
\end{array}\right.
$$

Secondly, we identify the following $k$-th Laplace fractional residual functions of (15) as:

$$
\begin{gathered}
\mathcal{L}\left\{\operatorname{Res}_{k}(x, s)\right\}=U_{k}(x, s)-\frac{\sinh x}{s} \\
-\frac{1}{s^{\beta}} D_{x} V_{k}(x, s)+\frac{1}{s^{\beta}} U_{k}(x, s)+\frac{1}{s^{\beta}} V_{k}(x, s), \\
\mathcal{L}\left\{\operatorname{Res}_{k}(x, s)\right\}=V_{k}(x, s)-\frac{\cosh x}{s} \\
-\frac{1}{s^{\beta}} D_{x} U_{k}(x, s)+\frac{1}{s^{\beta}} U_{k}(x, s)+\frac{1}{s^{\beta}} V_{k}(x, s) .
\end{gathered}
$$

where $U_{k}(x, s)$, and $V_{k}(x, s)$, represent the $k$-th Laplace series solutions of (15) and which are defined as:

$$
\begin{aligned}
& U_{k}(x, s)=\frac{\sinh x}{s}+\sum_{m=1}^{k} \frac{u_{m}(x)}{s^{m \beta+1}} s>0 \\
& V_{k}(x, s)=\frac{\cosh x}{s}+\sum_{m=1}^{i} \frac{v_{m}(x)}{s^{m \beta+1}} s>0
\end{aligned}
$$

Now, to find out the forms of $u_{1}(x)$, and $v_{1}(x)$, we write the $1^{\text {st }}$ - Laplace series solutions of (18) into the $1^{\text {st }}$ - Laplace fractional residual functions such that:

$$
\begin{aligned}
\mathcal{L}\left\{\operatorname{Res}_{1}(x, s)\right\} & =\frac{u_{1}(x)}{s^{\beta+1}} \\
& -\frac{1}{s^{\beta}} D_{x}\left(\frac{\cosh x}{s}+\frac{v_{1}(x)}{s^{\beta+1}}\right) \\
& +\frac{1}{s^{\beta}}\left(\frac{\sinh x}{s}+\frac{u_{1}(x)}{s^{\beta+1}}\right) \\
& +\frac{1}{s^{\beta}}\left(\frac{\cosh x}{s}+\frac{v_{1}(x)}{s^{\beta+1}}\right) \\
=\frac{u_{1}(x)}{s^{\beta+1}}-\frac{v_{1}^{\prime}(x)}{s^{2 \beta+1}}+\frac{u_{1}(x)}{s^{2 \beta+1}}+\frac{\cosh x}{s^{\beta+1}} & +\frac{v_{1}(x)}{s^{2 \beta+1}},
\end{aligned}
$$




$$
\begin{aligned}
& \mathcal{L}\left\{\operatorname{Res}_{1}(x, s)\right\}= \frac{u_{1}(x)}{s^{\beta+1}}-\frac{\cosh x}{s} \\
&-\frac{1}{s^{\beta}} D_{x}\left(\frac{\sinh x}{s}+\frac{u_{1}(x)}{s^{\beta+1}}\right) \\
&+\frac{1}{s^{\beta}}\left(\frac{\sinh x}{s}+\frac{u_{1}(x)}{s^{\beta+1}}\right) \\
&+\frac{1}{s^{\beta}}\left(\frac{\cosh x}{s}+\frac{v_{1}(x)}{s^{\beta+1}}\right) \\
&=\frac{v_{1}(x)}{s^{\beta+1}}-\frac{u_{1}^{\prime}(x)}{s^{2 \beta+1}}+\frac{u_{1}(x)}{s^{2 \beta+1}}+\frac{\sinh x}{s^{\beta+1}}+\frac{v_{1}(x)}{s^{2 \beta+1}} .
\end{aligned}
$$

Then, we multiply (18) by $s^{\beta+1}$, to get

$$
\begin{aligned}
s^{\beta+1} \mathcal{L}\left\{\operatorname{Res}_{1}(x, s)\right\} & \\
& =u_{1}(x)+\cosh x-\frac{v_{1}^{\prime}(x)}{s^{\beta+1}} \\
& +\frac{u_{1}(x)}{s^{\beta+1}}+\frac{v_{1}(x)}{s^{\beta+1}}
\end{aligned}
$$$$
s^{\beta+1} \mathcal{L}\left\{\operatorname{Res}_{1}(x, s)\right\}
$$$$
\begin{aligned}
& =v_{1}(x)+\sinh x-\frac{u_{1}^{\prime}(x)}{s^{\beta+1}} \\
& +\frac{u_{1}(x)}{s^{\beta+1}}+\frac{v_{1}(x)}{s^{\beta+1}} .
\end{aligned}
$$

Thereafter, by finding the limits of the obtained equation when $s \rightarrow \infty$, we conclude that $u_{1}(x)=$ $-\cosh x$, and $v_{1}(x)=-\sinh x$.

For $k=2$, the $2^{\text {nd }}$ - Laplace fractional residual functions could be written as:

$$
\begin{gathered}
\mathcal{L}\left\{\operatorname{Res}_{2}(x, s)\right\}=U_{2}(x, s)-\frac{\sinh x}{s}-\frac{1}{s^{\beta}} D_{x} V_{2}(x, s) \\
+\frac{1}{s^{\beta}} U_{2}(x, s)+\frac{1}{s^{\beta}} V_{2}(x, s) \\
\mathcal{L}\left\{\operatorname{Res}_{2}(x, s)\right\}=V_{2}(x, s)-\frac{\cosh x}{s}-\frac{1}{s^{\beta}} D_{x} U_{2}(x, s) \\
+\frac{1}{s^{\beta}} U_{2}(x, s)+\frac{1}{s^{\beta}} V_{2}(x, s) .
\end{gathered}
$$

Where

$$
\begin{aligned}
U_{2}(x, s) & =\frac{\sinh x}{s}-\frac{\cosh x}{s^{\beta+1}}+\frac{u_{2}(x)}{s^{2 \beta+1}}, \\
\text { And } \quad V_{2}(x, s) & =\frac{\cosh x}{s}-\frac{\sinh x}{s^{\beta+1}}+\frac{v_{2}(x)}{s^{2 \beta+1}} .
\end{aligned}
$$

Thus, we have

$$
\begin{aligned}
\mathcal{L}\left\{\operatorname{Res}_{2}(x, s)\right\} & =-\frac{\cosh x}{s^{\beta+1}}+\frac{u_{2}(x)}{s^{2 \beta+1}} \\
& -\frac{v_{2}^{\prime}(x)}{s^{3 \beta+1}}+\frac{u_{2}(x)}{s^{3 \beta+1}}+\frac{\cosh x}{s^{\beta+1}} \\
& -\frac{\sinh x}{s^{2 \beta+1}}+\frac{v_{2}(x)}{s^{3 \beta+1}}
\end{aligned}
$$

$$
\begin{aligned}
\mathcal{L}\left\{\operatorname{Res}_{2}(x, s)\right\} & =-\frac{\sinh x}{s^{\beta+1}}+\frac{v_{2}(x)}{s^{2 \beta+1}}-\frac{u_{2}^{\prime}(x)}{s^{3 \beta+1}} \\
& +\frac{u_{2}(x)}{s^{3 \beta+1}}+\frac{\cosh x}{s^{\beta+1}}-\frac{\sinh x}{s^{2 \beta+1}} \\
& +\frac{v_{2}(x)}{s^{3 \beta+1}} .
\end{aligned}
$$

Consequently, multiply (21) by $s^{2 \beta+1}$ to get:

$$
\begin{aligned}
s^{2 \beta+1} \mathcal{L}\left\{\operatorname{Res}_{2}(x, s)\right\} & u_{2}(x)-\frac{v_{2}^{\prime}(x)}{s^{\beta}}+\frac{u_{2}(x)}{s^{\beta}} \\
& -\sinh x+\frac{v_{2}(x)}{s^{\beta}}, \\
s^{2 \beta+1} \mathcal{L}\left\{\operatorname{Res}_{2}(\right. & x, s)\} \\
& =v_{2}(x)-\frac{u_{2}^{\prime}(x)}{s^{\beta}}+\frac{u_{2}(x)}{s^{\beta}} \\
& -\cosh x+\frac{v_{2}(x)}{s^{\beta}} .
\end{aligned}
$$

Depending on the results

$$
\begin{aligned}
& \lim _{s \rightarrow \infty} s^{2 \beta+1} \mathcal{L}\left\{\operatorname{Res}_{2}(x, s)\right\}=0, \text { and } \\
& \lim _{s \rightarrow \infty} s^{2 \beta+1} \mathcal{L}\left\{\operatorname{Res}_{2}(x, s)\right\}=0,
\end{aligned}
$$

gives $u_{2}(x)=\sinh x$, and $v_{2}(x)=\cosh x$.

For $k=3$, substitute $U_{3}(x, s)=\frac{\sinh x}{s}-\frac{\cosh x}{s^{\beta+1}}+$ $\frac{\sinh x}{s^{2 \beta+1}}+\frac{u_{3}(x)}{s^{3 \beta+1}} \quad$ and $\quad V_{3}(x, s)=\frac{\cosh x}{s}-\frac{\sinh x}{s^{\beta+1}}+$ $\frac{\cosh x}{s^{2 \beta+1}}+\frac{v_{3}(x)}{s^{3 \beta+1}}$ into $\mathcal{L}\left\{\operatorname{Res}_{3}(x, s)\right\}, \mathcal{L}\left\{\operatorname{Res}_{3}(x, s)\right\}$ of (16) so that

$$
\begin{aligned}
\mathcal{L}\left\{\operatorname{Res}_{3}(x, s)\right\} & =\frac{u_{3}(x)}{s^{3 \beta+1}}-\frac{v_{3}^{\prime}(x)}{s^{4 \beta+1}}+\frac{u_{3}(x)}{s^{4 \beta+1}} \\
& +\frac{\cosh x}{s^{3 \beta+1}}+\frac{u_{3}(x)}{s^{4 \beta+1}}, \\
\mathcal{L}\left\{\operatorname{Res}_{3}(x, s)\right\} & =\frac{v_{3}(x)}{s^{3 \beta+1}}-\frac{v_{3}^{\prime}(x)}{s^{4 \beta+1}}+\frac{\sinh x}{s^{3 \beta+1}} \\
& +\frac{u_{3}(x)}{s^{4 \beta+1}}+\frac{u_{3}(x)}{s^{4 \beta+1}} .
\end{aligned}
$$

Then, multiply (23) by $s^{3 \beta+1}$ to get:

$$
\begin{aligned}
s^{3 \beta+1} \mathcal{L}\left\{\operatorname{Res}_{3}(x, s)\right\} & u_{3}(x)-\frac{v_{3}^{\prime}(x)}{s^{\beta}}+\frac{u_{3}(x)}{s^{\beta}} \\
& +\cosh x+\frac{u_{3}(x)}{s^{\beta}}, \\
s^{3 \beta+1} \mathcal{L}\left\{\operatorname{Res}_{3}(x, s)\right\} & \\
& =v_{3}(x)-\frac{v_{3}^{\prime}(x)}{s^{\beta}}+\sinh x \\
& +\frac{u_{3}(x)}{s^{\beta}}+\frac{u_{3}(x)}{s^{\beta}} .
\end{aligned}
$$

Depending on the results

$\lim _{s \rightarrow \infty} s^{3 \beta+1} \mathcal{L}\left\{\operatorname{Res}_{3}(x, s)\right\}=0$ and

$\lim _{s \rightarrow \infty} s^{3 \beta+1} \mathcal{L}\left\{\operatorname{Res} V_{3}(x, s)\right\}=0$, gives $u_{3}(x)=$ $-\cosh x$, and $v_{3}(x)=-\sinh x$. 
Continue with the same argument and based on the results of $\lim _{s \rightarrow \infty} s^{k \beta+1} \mathcal{L}\left\{\operatorname{Res} U_{k}(x, s)\right\}=0, \quad$ and $\lim _{s \rightarrow \infty} s^{k \beta+1} \mathcal{L}\left\{\operatorname{Res}_{k}(x, s)\right\}=0$, for $k=4,5,6, \ldots$, it yields:

$u_{4}(x)=\sinh x, \quad v_{4}(x)=\cosh x$

$u_{5}(x)=\quad v_{5}(x)=$

$-\cosh x, \quad-\sinh x$.

$u_{6}(x)=\sinh x, \quad v_{6}(x)=\cosh x$,

Therefore, the Laplace series solutions of (15) could be written as:

$$
\begin{aligned}
& \begin{aligned}
& U(x, s)=\frac{\sinh x}{s}-\frac{\cosh x}{s^{\beta+1}}+\frac{\sinh x}{s^{2 \beta+1}} \\
&-\frac{\cosh x}{s^{3 \beta+1}}+\frac{\sinh x}{s^{4 \beta+1}}+\cdots, \\
& V(x, s)=\frac{\cosh x}{s}-\frac{\sinh x}{s^{\beta+1}}+\frac{\cosh x}{s^{2 \beta+1}} \\
&-\frac{\sinh x}{s^{3 \beta+1}}+\frac{\cosh x}{s^{4 \beta+1}}+\cdots .
\end{aligned}
\end{aligned}
$$

Finally, we apply the inverse transform on both sides of (25) to get the following multiple fractional PS approximate solutions for the original system (14) as:

$u(x, t)$

$=\sinh x \sum_{m=0}^{\infty} \frac{t^{2 m \beta}}{\Gamma(2 m \beta+1)}$
$-\cosh x \sum_{m=0}^{\infty} \frac{t^{(2 m+1) \beta}}{\Gamma((2 m+1) \beta+1)^{\prime}}$

$$
\begin{aligned}
& v(x, t) \\
& =\cosh x \sum_{m=0}^{\infty} \frac{t^{2 m \beta}}{\Gamma(2 m \beta+1)} \\
& -\sinh x \sum_{m=0}^{\infty} \frac{t^{(2 m+1) \beta}}{\Gamma((2 m+1) \beta+1)}
\end{aligned}
$$

which are coinciding with the McLaurin expansion series of the exact solutions $u(x, t)=\sinh (x-t)$ and $v(x, t)=\cosh (x-t)$ when $\beta=1$. Further, the results are the same as in [36].

Numerical simulation of the exact and tenth multiple fractional PS approximate solutions are performed for Example 4.1, at $\beta=1$, for some selected grid points with step size 0.2 on the interval $[0,1]$ that shown in Table 1. Graphically, the tenth multiple fractional PS approximate solutions versus the exact solutions for system (14) is plotted in 3dimensional space for $t \in[0,1]$, and $x \in[-2,2]$,

\begin{tabular}{|c|c|c|c|c|}
\hline $\boldsymbol{x}_{\boldsymbol{i}}$ & $t_{i}$ & $u(x, t)$ & $u_{10}(x, t)$ & $\left|u(x, t)-u_{10}(x, t)\right|$ \\
\hline \multirow{6}{*}{0} & 0.00 & 0.0 & 0.0 & 0.0 \\
\hline & 0.20 & -0.2013360025410940 & -0.2013360025410935 & $4.996003610813204 \times 10^{-16}$ \\
\hline & 0.40 & -0.4107523258028155 & -0.4107523258017637 & $1.051825293529873 \times 10^{-12}$ \\
\hline & 0.60 & -0.6366535821482414 & -0.6366535820 & $9.109846210719752 \times 10^{-11}$ \\
\hline & 0.80 & -0.8881059821 & 81059800 & 2.160815193441578 \\
\hline & 1.00 & -1 . & 116 & 66203766 \\
\hline \multirow{6}{*}{1} & 0.00 & & 1. & 0.00 \\
\hline & 0.20 & 0.88810598218 & 0.88810598218762380 & $7.771561172376096 \times 10^{-16}$ \\
\hline & 0.40 & 0.63665358214824130 & 0.63665358214 & $1.581956787788385 \times 10^{-14}$ \\
\hline & 0.60 & 0 & 32 & $1.352212786187578 \times 10^{-12}$ \\
\hline & 0.80 & 6002 & 0.2 & 3.1651 \\
\hline & 1.00 & 0.00 & $3.6439436135183 \times 10^{-8}$ & $3.6439436135182740 \times 10^{-8}$ \\
\hline$x_{i}$ & $t_{i}$ & $v(x, t)$ & $v_{10}(x, t)$ & $\left|v(x, t)-v_{10}(x, t)\right|$ \\
\hline \multirow{5}{*}{0} & 0.00 & & & 0.00 \\
\hline & 0.20 & 6755 & 66755 & 0.00 \\
\hline & 0.40 & 1.0810723718384547 & 1.0810723718384199 & 3.4861002973229 \\
\hline & 0.60 & 1.1854652182422678 & 1.1854652182377146 & $4.553246668592692 \times 10^{-12}$ \\
\hline & 0.80 & 1.3374349463048447 & 1.337434946160875 & $1.43969725030502 \times 10^{-10}$ \\
\hline
\end{tabular}
when $\beta=1$, as in Fig.1. Notice that the pattern of the exact solutions consistent and in good agreement with the pattern of the approximated solutions in their domains, which confirms the effectiveness and performance of the LRPS method.

Table 1. Numerical results at $\beta=1$ and $n=10$ with different values of $t$ of Example 4.1. 


\begin{tabular}{ccccc} 
& 1.00 & 1.5430806348152437 & 1.5430806327160496 & $2.099194151838901 \times 10^{-9}$ \\
\hline \multirow{3}{*}{0.00} & 1.5430806348152437 & 1.5430806348152437 & 0.00 \\
& 0.20 & 1.3374349463048447 & 1.3374349463048452 & $4.440892098500626 \times 10^{-16}$ \\
1 & 0.40 & 1.1854652182422676 & 1.1854652182434500 & $1.182387521225791 \times 10^{-12}$ \\
& 0.60 & 1.0810723718384547 & 1.0810723719384878 & $1.000330929201709 \times 10^{-10}$ \\
& 0.80 & 1.0200667556190760 & 1.0200667579363119 & $2.317235958670949 \times 10^{-9}$ \\
& 1.00 & 1.00 & 1.0000000263916695 & $2.639166951645677 \times 10^{-8}$ \\
\hline
\end{tabular}

(a)

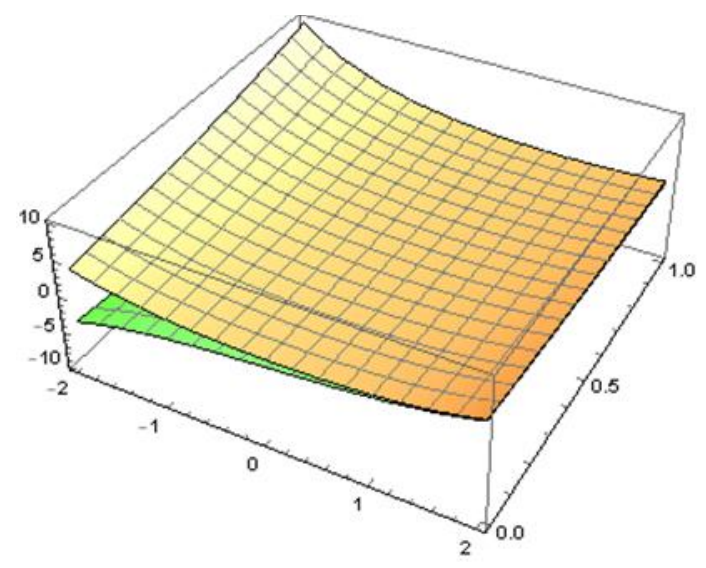

$Z Z Z u(x, t) \quad X \times v(x, t)$

(b)

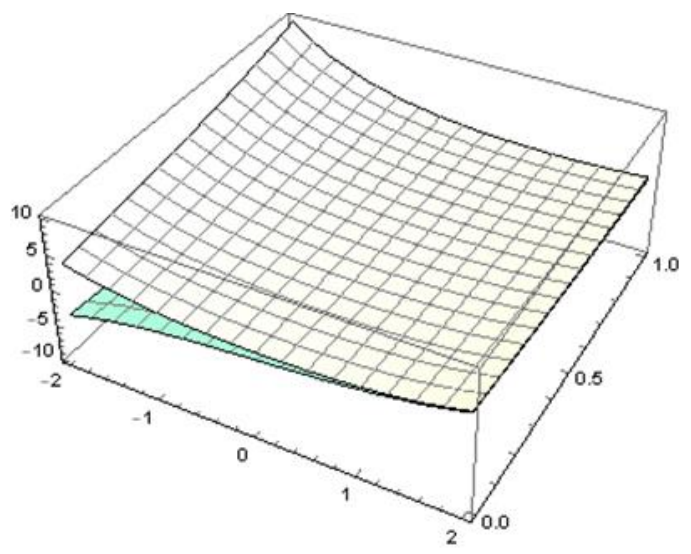

$Z z-u_{10}(x, t) \curvearrowright v_{10}(x, t)$

Fig. 1 (a) 3D-Surfaces Plot of Exact solutions $u(x, t)$ and $v(x, t)$ at $\beta=1$; (b) 3D-Surfaces Plot of tenth multiple fractional PS approximate solutions $u_{10}(x, t)$ and $v_{10}(x, t)$ at $\beta=1$ for Example 4.1.

Example 4.2: Consider the following non-linear system of FIVPs [36,37]:

$$
\left\{\begin{array}{c}
\mathfrak{D}_{t}^{\beta} u+D_{x}^{2} u+2 u D_{x} u+D_{x}(u v)=0 \\
\mathfrak{D}_{t}^{\beta} v+D_{x}^{2} v+2 v D_{x} v+D_{x}(u v)=0, \\
u(x, 0)=e^{x}, v(x, 0)=-e^{x} \\
\text { where } x \in R, t \geq 0, \beta \in(0,1] .
\end{array}\right.
$$

For $\beta=1$, the exact solutions of (27) are $u(x, t)=$ $e^{x-t}$, and $v(x, t)=-e^{x-t}$.

According to the Laplace RPS approach, we employ the Laplace transform to both sides of (27), we can get the following Laplace system:

$U(x, s)$

$=\frac{e^{x}}{s}-\frac{1}{s^{\beta}} D_{x}^{2} U(x, s)$

$-\frac{2}{s^{\beta}} \mathcal{L}\left\{\left(\mathcal{L}^{-1} U(x, s)\right)\left(\mathcal{L}^{-1} D_{x} U(x, s)\right)\right\}$

$-\frac{1}{s^{\beta}} D_{x} \mathcal{L}\left\{\left(\mathcal{L}^{-1} U(x, s)\right)\left(\mathcal{L}^{-1} V(x, s)\right)\right\}$,

$V(x, s)$

$=\frac{e^{x}}{s}-\frac{1}{s^{\beta}} D_{x}^{2} V(x, s)$

$-\frac{2}{s^{\beta}} \mathcal{L}\left\{\left(\mathcal{L}^{-1} V(x, s)\right)\left(\mathcal{L}^{-1} D_{x} V(x, s)\right)\right\}$

$-\frac{1}{s^{\beta}} D_{x} \mathcal{L}\left\{\left(\mathcal{L}^{-1} V(x, s)\right)\left(\mathcal{L}^{-1} V(x, s)\right)\right\}$.

Suppose that the $k$-th Laplace series solutions of (28) have the forms:

$$
\begin{array}{cc}
U_{k}(x, s)=\frac{e^{x}}{s}+\sum_{m=1}^{k} \frac{u_{m}(x)}{s^{m \beta+1}}, & s>0, \\
V_{k}(x, s)=-\frac{e^{x}}{s}+\sum_{m=1}^{k} \frac{v_{m}(x)}{s^{m \beta+1}}, \quad s>0 .
\end{array}
$$

Consequently, the $k$-th Laplace fractional residual functions can be reformulated as: 


$$
\begin{aligned}
\mathcal{L}\left\{\operatorname{Res}_{k}(x, s)\right\} & =\sum_{m=1}^{k} \frac{u_{m}(x)}{s^{m \beta+1}}+\frac{1}{s^{\beta}} D_{x}^{2}\left(\frac{e^{x}}{s}+\sum_{m=1}^{k} \frac{u_{m}(x)}{s^{m \beta+1}}\right) \\
& +\frac{2}{s^{\beta}} \mathcal{L}\left\{\left(\mathcal{L}^{-1}\left(\frac{e^{x}}{s}+\sum_{m=1}^{k} \frac{u_{m}(x)}{s^{m \beta+1}}\right)\right)\left(\mathcal{L}^{-1} D_{x}\left(\frac{e^{x}}{s}+\sum_{m=1}^{k} \frac{u_{m}(x)}{s^{m \beta+1}}\right)\right)\right\} \\
& +\frac{1}{s^{\beta}} D_{x} \mathcal{L}\left\{\left(\mathcal{L}^{-1}\left(\frac{e^{x}}{s}+\sum_{m=1}^{k} \frac{u_{m}(x)}{s^{m \beta+1}}\right)\right)\left(\mathcal{L}^{-1}\left(-\frac{e^{x}}{s}+\sum_{m=1}^{k} \frac{v_{m}(x)}{s^{m \beta+1}}\right)\right)\right\},
\end{aligned}
$$

$\mathcal{L}\left\{\operatorname{Res}_{k}(x, s)\right\}=$

$$
\begin{aligned}
& =\sum_{m=1}^{k} \frac{v_{m}(x)}{s^{m \beta+1}}+\frac{1}{s^{\beta}} D_{x}^{2}\left(-\frac{e^{x}}{s}+\sum_{m=1}^{k} \frac{v_{m}(x)}{s^{m \beta+1}}\right) \\
& +\frac{2}{s^{\beta}} \mathcal{L}\left\{\left(\mathcal{L}^{-1}\left(-\frac{e^{x}}{s}+\sum_{m=1}^{k} \frac{v(x)}{s^{m \beta+1}}\right)\right)\left(\mathcal{L}^{-1} D_{x}\left(-\frac{e^{x}}{s}+\sum_{m=1}^{k} \frac{v_{m}(x)}{s^{m \beta+1}}\right)\right)\right\} \\
& +\frac{1}{s^{\beta}} D_{x} \mathcal{L}\left\{\left(\mathcal{L}^{-1}\left(-\frac{e^{x}}{s}+\sum_{m=1}^{k} \frac{v_{m}(x)}{s^{m \beta+1}}\right)\right)\left(\mathcal{L}^{-1}\left(\frac{e^{x}}{s}+\sum_{m=1}^{k} \frac{u_{m}(x)}{s^{m \beta+1}}\right)\right)\right\} .
\end{aligned}
$$

For $k=1$, the $1^{\text {st }}$ - Laplace fractional residual functions will be as:

$$
\begin{aligned}
\mathcal{L}\left\{\operatorname{Res}_{1}(x, s)\right\} & =\frac{u_{1}(x)}{s^{\beta+1}}+\frac{1}{s^{\beta}}\left(\frac{e^{x}}{s}+\frac{u_{1}^{\prime \prime}(x)}{s^{\beta+1}}\right)+\frac{2}{s^{\beta}} \mathcal{L}\left\{\left(\mathcal{L}^{-1}\left(\frac{e^{x}}{s}+\frac{u_{1}(x)}{s^{\beta+1}}\right)\right)\left(\mathcal{L}^{-1}\left(\frac{e^{x}}{s}+\frac{u_{1}^{\prime}(x)}{s^{\beta+1}}\right)\right)\right\} \\
& +\frac{1}{s^{\beta}} D_{x} \mathcal{L}\left\{\left(\mathcal{L}^{-1}\left(\frac{e^{x}}{s}+\frac{u_{1}(x)}{s^{\beta+1}}\right)\right)\left(\mathcal{L}^{-1}\left(-\frac{e^{x}}{s}+\frac{v_{1}(x)}{s^{\beta+1}}\right)\right)\right\} \\
\mathcal{L}\left\{\operatorname{Res}_{1}(x, s)\right\} & =\frac{v_{1}(x)}{s^{\beta+1}}+\frac{1}{s^{\beta}} D_{x}^{2}\left(-\frac{e^{x}}{s}+\frac{v_{1}^{\prime \prime}(x)}{s^{\beta+1}}\right) \\
& +\frac{2}{s^{\beta}} \mathcal{L}\left\{\left(\mathcal{L}^{-1}\left(-\frac{e^{x}}{s}+\frac{v_{1}(x)}{s^{\beta+1}}\right)\right)\left(\mathcal{L}^{-1}\left(-\frac{e^{x}}{s}+\frac{v_{1}^{\prime}(x)}{s^{\beta+1}}\right)\right)\right\} \\
& +\frac{1}{s^{\beta}} D_{x} \mathcal{L}\left\{\left(\mathcal{L}^{-1}\left(-\frac{e^{x}}{s}+\frac{v_{1}(x)}{s^{\beta+1}}\right)\right)\left(\mathcal{L}^{-1}\left(\frac{e^{x}}{s}+\frac{u_{1}(x)}{s^{\beta+1}}\right)\right)\right\} .
\end{aligned}
$$

Multiplication the obtained equations by $s^{\beta+1}$, we

get

$$
\begin{aligned}
& s^{\beta+1} \mathcal{L}\left\{\operatorname{Res} U_{1}(x, s)\right\} \\
& =e^{x}+u_{1}(x)+e^{x} \frac{u_{1}(x)}{s^{\beta}}+e^{x} \frac{v_{1}(x)}{s^{\beta}}+e^{x} \frac{u_{1}^{\prime}(x)}{s^{\beta}}+\frac{2 \Gamma(2 \beta+1) u_{1}(x) u_{1}^{\prime}(x)}{\Gamma^{2}(\beta+1) s^{2 \beta}} \\
& +\frac{\Gamma(2 \beta+1) v_{1}(x) u_{1}^{\prime}(x)}{s^{2 \beta} \Gamma^{2}(\beta+1)}+e^{x} \frac{v_{1}^{\prime}(x)}{s^{\beta}}+\frac{\Gamma(2 \beta+1) u_{1}(x) v_{1}^{\prime}(x)}{\Gamma^{2}(\beta+1) s^{2 \beta}}+\frac{u_{1}^{\prime \prime}(x)}{s^{\beta}} \\
& s^{\beta+1} \mathcal{L}\left\{\operatorname{Res}_{1}(x, s)\right\}= \\
& =-e^{x}+v_{1}(x)-e^{x} \frac{u_{1}(x)}{s^{\beta}}-e^{x} \frac{v_{1}(x)}{s^{\beta}}-e^{x} \frac{u_{1}^{\prime}(x)}{s^{\beta}}+\frac{\Gamma(2 \beta+1) v_{1}(x) u_{1}^{\prime}(x)}{s^{2 \beta} \Gamma^{2}(\beta+1)} \\
& -e^{x} \frac{v_{1}^{\prime}(x)}{s^{\beta}}+\frac{\Gamma(2 \beta+1) u_{1}(x) v_{1}^{\prime}(x)}{s^{2 \beta} \Gamma^{2}(\beta+1)}+\frac{2 \Gamma(2 \beta+1) u_{1}(x) v_{1}^{\prime}(x)}{\Gamma^{2}(\beta+1) s^{2 \beta}}+\frac{v_{1}^{\prime \prime}(x)}{s^{\beta}} \text {. }
\end{aligned}
$$


and by solving the systems

$\lim _{s \rightarrow \infty} s^{\beta+1} \mathcal{L}\left\{\operatorname{Res}_{1}(x, s)\right\}=0$, and

$\lim _{s \rightarrow \infty} s^{\beta+1} \mathcal{L}\left\{\operatorname{Res}_{1}(x, s)\right\}=0$, gives $u_{1}(x)=$ $-e^{x}$, and $v_{1}(x)=e^{x}$.

For $k=2$, we have

$$
\begin{aligned}
s^{2 \beta+1} \mathcal{L}\left\{\operatorname{Res}_{2}(\right. & x, s)\} \\
& =e^{x}+u_{2}(x)+e^{x} \frac{u_{2}(x)}{s^{\beta}} \\
& +e^{x} \frac{v_{2}(x)}{s^{\beta}}+e^{x} \frac{u_{2}^{\prime}(x)}{s^{\beta}} \\
& +\frac{e^{x} \Gamma(3 \beta+1) u_{2}(x)}{\Gamma(\beta+1) \Gamma(2 \beta+1) s^{2 \beta}} \\
& -\frac{e^{x} \Gamma(3 \beta+1) v_{2}(x)}{\Gamma(\beta+1) \Gamma(2 \beta+1) s^{2 \beta}} \\
& +e^{x} \frac{v_{2}^{\prime}(x)}{s^{\beta}} \\
& -\frac{e^{x} \Gamma(3 \beta+1) u_{2}^{\prime}(x)}{\Gamma(\beta+1) \Gamma(2 \beta+1) s^{2 \beta}} \\
& -\frac{e^{x} \Gamma(3 \beta+1) v_{2}^{\prime}(x)}{\Gamma(\beta+1) \Gamma(2 \beta+1) s^{2 \beta}} \\
& +\frac{\Gamma(4 \beta+1) v_{2}(x) u_{2}^{\prime}(x)}{\Gamma^{2}(2 \beta+1) s^{3 \beta}} \\
& +\frac{\Gamma(4 \beta+1) u_{2}(x) v_{2}^{\prime}(x)}{\Gamma^{2}(2 \beta+1) s^{3 \beta}}+\frac{u_{2}^{\prime \prime}(x)}{s^{\beta}}
\end{aligned}
$$

$s^{2 \beta+1} \mathcal{L}\left\{\operatorname{Res}_{2}(x, s)\right\}$

$$
\begin{aligned}
& =e^{x}+v_{2}(x)-e^{x} \frac{u_{2}(x)}{s^{\beta}} \\
& -e^{x} \frac{v_{2}(x)}{s^{\beta}}-e^{x} \frac{u_{2}^{\prime}(x)}{s^{\beta}} \\
& +\frac{e^{x} \Gamma(3 \beta+1) u_{2}(x)}{\Gamma(\beta+1) \Gamma(2 \beta+1) s^{2 \beta}} \\
& +\frac{e^{x} \Gamma(3 \beta+1) v_{2}(x)}{\Gamma(\beta+1) \Gamma(2 \beta+1) s^{2 \beta}} \\
& -e^{x} \frac{v_{2}^{\prime}(x)}{s^{\beta}} \\
& +\frac{e^{x} \Gamma(3 \beta+1) u_{2}^{\prime}(x)}{\Gamma(\beta+1) \Gamma(2 \beta+1) s^{2 \beta}} \\
& +\frac{e^{x} \Gamma(3 \beta+1) v_{2}^{\prime}(x)}{\Gamma(\beta+1) \Gamma(2 \beta+1) s^{2 \beta}} \\
& +\frac{\Gamma(4 \beta+1) v_{2}(x) u_{2}^{\prime}(x)}{\Gamma^{2}(2 \beta+1) s^{3 \beta}} \\
& +\frac{\Gamma(4 \beta+1) u_{2}(x) v_{2}^{\prime}(x)}{\Gamma^{2}(2 \beta+1) s^{3 \beta}}+\frac{v_{2}^{\prime \prime}(x)}{s^{\beta}}
\end{aligned}
$$

Depending on the fact $\lim _{s \rightarrow \infty} s^{2 \beta+1} \mathcal{L}\left\{\operatorname{Res} U_{2}(x, s)\right\}=$ 0 , and $\lim _{s \rightarrow \infty} s^{2 \beta+1} \mathcal{L}\left\{\operatorname{ResV}_{2}(x, s)\right\}=0$, we get $u_{2}(x) \stackrel{s \rightarrow \infty}{=} e^{x}$, and $v_{2}(x)=-e^{x}$.

Following the procedure of Laplace RPS method, the unknown functions in the expansions (29) can be obtained by solving the system,

$\lim _{s \rightarrow \infty} s^{k \beta+1} \mathcal{L}\left\{\operatorname{Res} U_{k}(x, s)\right\}=0$, and

$\lim _{s \rightarrow \infty} s^{k \beta+1} \mathcal{L}\left\{\operatorname{Res}_{k}(x, s)\right\}=0$, for $u_{k}(x)$, and $v_{k}(x)$. Hence, the Laplace series solutions of (28) can be given as:

$$
\begin{aligned}
U(x, s)=\frac{e^{x}}{s}- & \frac{e^{x}}{s^{\beta+1}}+\frac{e^{x}}{s^{2 \beta+1}}-\frac{e^{x}}{s^{3 \beta+1}} \\
& +\frac{e^{x}}{s^{4 \beta+1}}-\frac{e^{x^{5}}}{s^{5 \beta+1}}+\cdots \\
& =e^{x} \sum_{m=0}^{\infty} \frac{(-1)^{m}}{s^{m \beta+1}}, \\
V(x, s)=-\frac{e^{x}}{s}+ & \frac{e^{x}}{s^{\beta+1}}-\frac{e^{x}}{s^{2 \beta+1}}+\frac{e^{x}}{s^{3 \beta+1}} \\
& -\frac{e^{x}}{s^{4 \beta+1}}+\frac{e^{x}}{s^{5 \beta+1}}+\cdots \\
= & -e^{x} \sum_{m=0}^{\infty} \frac{(-1)^{m}}{s^{m \beta+1}},
\end{aligned}
$$

Finally, we operate the inverse Laplace transform to the Laplace series solutions (34), to conclude that the multiple fractional PS approximate solutions for the IVPs system (27) can be expressed as:

$$
\begin{aligned}
u(x, t)=e^{x}( & 1-\frac{t^{\beta}}{\Gamma(\beta+1)}+\frac{t^{2 \beta}}{\Gamma(2 \beta+1)} \\
& -\frac{t^{3 \beta}}{\Gamma(3 \beta+1)}+\frac{t^{4 \beta}}{\Gamma(4 \beta+1)} \\
& +\cdots), \\
v(x, t)=-e^{x}( & 1-\frac{t^{\beta}}{\Gamma(\beta+1)}+\frac{t^{2 \beta}}{\Gamma(2 \beta+1)} \\
& -\frac{t^{3 \beta}}{\Gamma(3 \beta+1)}+\frac{t^{4 \beta}}{\Gamma(4 \beta+1)} \\
& +\cdots)
\end{aligned}
$$

If we substitute $\beta=1$, then the multiple fractional PS solutions (35) reduce to

$$
u(x, t)=e^{x}\left(1-t+\frac{t^{2}}{2 !}-\frac{t^{3}}{3 !}+\frac{t^{4}}{4 !}+\cdots\right) .
$$




$$
\begin{gathered}
v(x, t)=-e^{x}\left(1-t+\frac{t^{2}}{2 !}-\frac{t^{3}}{3 !}+\frac{t^{4}}{4 !}\right. \\
+\cdots) .
\end{gathered}
$$

which agree with McLaurin series expansions of the exact solutions $u(x, t)=e^{x-t}$, and $v(x, t)=$ $-e^{x-t}$. Further, the results are the same as in [36] and [37].

Graphically, to demonstrate the impact of parameters $\beta$, on the behavior solutions, we plot the exact and tenth approximate solutions for Example 4.2 that shown in Fig.2. Further, numerical comparisons are performed to validate the accuracy of our approach by establishing the recurrence errors for the obtained approximate solutions of the IVPs system (27) at various values of $\beta$, and fixed value of $x=0$ as in Table 2 .

(a)

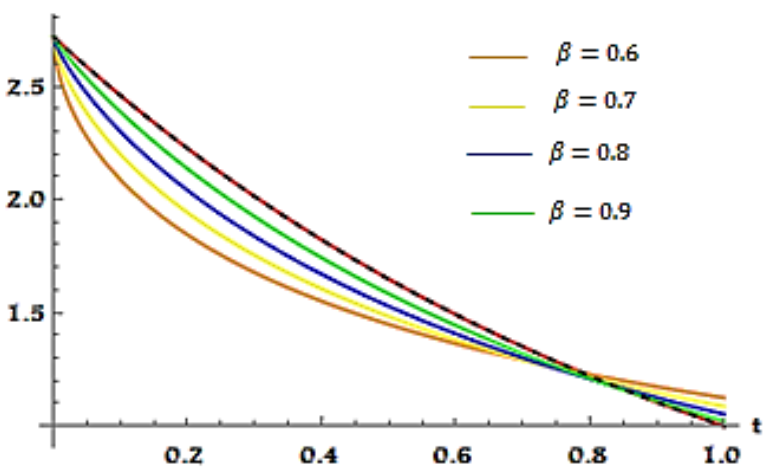

(b)

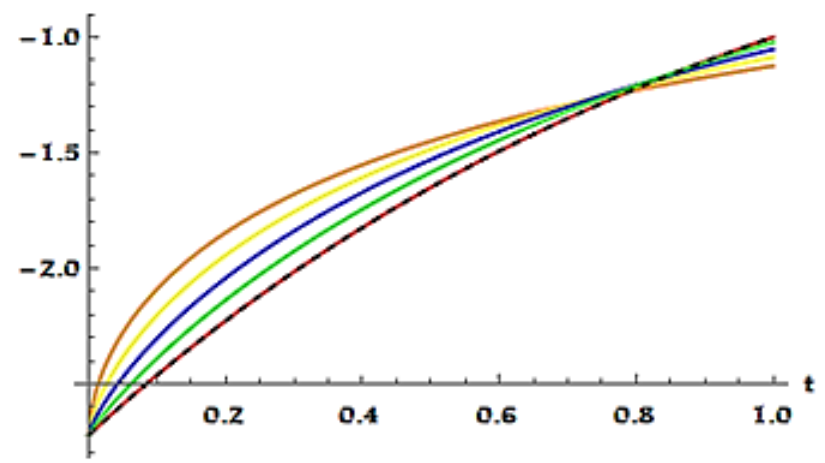

Fig.2 (a) Plots of exact $u(x, t)$, and $10^{\text {th }}$ approximate solution $u_{10}(x, t)$, at various values of $\beta$ and $x=0$, (b) Plots of exact $v(x, t)$, and $10^{\text {th }}$ approximate solution $v_{10}(x, t)$ at various values of $\beta$ and $x=0$.

of our approach by establishing the recurrence errors for the obtained approximate solutions of the IVPs system (27) at various values of $\beta$, and fixed value of $x=0$ as in Table 2 .

\begin{tabular}{ccccc}
\hline \multicolumn{5}{c}{$\left|\boldsymbol{u}_{\mathbf{8}}(\boldsymbol{x}, \boldsymbol{t})-\boldsymbol{u}_{\mathbf{7}}(\boldsymbol{x}, \boldsymbol{t})\right|$} \\
\hline $\boldsymbol{t}_{\boldsymbol{i}}$ & $\boldsymbol{\beta}=\mathbf{0 . 7}$ & $\boldsymbol{\beta}=\mathbf{0 . 8}$ & $\boldsymbol{\beta}=\mathbf{0 . 9}$ & $\boldsymbol{\beta}=\mathbf{1 . 0 0}$ \\
\hline 0.15 & $7.057767437 \times 10^{-8}$ & $3.460084330 \times 10^{-9}$ & $1.545980012 \times 10^{-10}$ & $6.356470905 \times 10^{-12}$ \\
0.30 & $3.423224005 \times 10^{-6}$ & $2.921989519 \times 10^{-7}$ & $2.273106625 \times 10^{-8}$ & $1.627232349 \times 10^{-9}$ \\
0.45 & $3.315479673 \times 10^{-5}$ & $3.914377550 \times 10^{-6}$ & $4.211888542 \times 10^{-7}$ & $4.170417999 \times 10^{-8}$ \\
0.60 & $1.660363947 \times 10^{-4}$ & $2.467576504 \times 10^{-5}$ & $3.342225695 \times 10^{-6}$ & $4.165714286 \times 10^{-7}$ \\
0.75 & $5.792944496 \times 10^{-4}$ & $1.029187989 \times 10^{-4}$ & $1.666433039 \times 10^{-5}$ & $2.482959202 \times 10^{-6}$ \\
0.90 & $1.608104789 \times 10^{-3}$ & $3.305633372 \times 10^{-4}$ & $6.1928824850 \times 10^{-5}$ & $1.067627009 \times 10^{-7}$ \\
\hline \multicolumn{5}{c}{$\left|\boldsymbol{v}_{\mathbf{8}}(\boldsymbol{x}, \boldsymbol{t})-\boldsymbol{v}_{\mathbf{7}}(\boldsymbol{x}, \boldsymbol{t})\right|$} \\
\hline $\boldsymbol{t}_{\boldsymbol{i}}$ & $\boldsymbol{\beta}=\mathbf{0 . 7}$ & $\boldsymbol{\beta}=\mathbf{0 . 8}$ & $\boldsymbol{\beta}=\mathbf{0 . 9}$ & $\boldsymbol{\beta}=\mathbf{1 . 0 0}$ \\
\hline 0.15 & $7.057767437 \times 10^{-8}$ & $3.460084330 \times 10^{-9}$ & $1.545980012 \times 10^{-10}$ & $6.356470905 \times 10^{-12}$ \\
0.30 & $3.423224005 \times 10^{-6}$ & $2.921989519 \times 10^{-7}$ & $2.273106625 \times 10^{-8}$ & $1.627232349 \times 10^{-9}$ \\
0.45 & $3.315479673 \times 10^{-5}$ & $3.914377550 \times 10^{-6}$ & $4.211888542 \times 10^{-7}$ & $4.170417999 \times 10^{-8}$ \\
0.60 & $1.660363947 \times 10^{-4}$ & $2.467576504 \times 10^{-5}$ & $3.342225695 \times 10^{-6}$ & $4.165714286 \times 10^{-7}$ \\
0.75 & $5.792944496 \times 10^{-4}$ & $1.029187989 \times 10^{-4}$ & $1.666433039 \times 10^{-5}$ & $2.482959202 \times 10^{-6}$ \\
0.90 & $1.608104789 \times 10^{-3}$ & $3.305633372 \times 10^{-4}$ & $6.1928824850 \times 10^{-5}$ & $1.067627009 \times 10^{-7}$ \\
\hline
\end{tabular}

Example 4.3: Consider the following non-linear system of FIVPs [36]: 


$$
\left\{\begin{array}{c}
\mathfrak{D}_{t}^{\beta} u-D_{x}^{2} u+u D_{x} u-u v=0 \\
\mathfrak{D}_{t}^{\beta} v-D_{x}^{2} v+v D_{x} v+u v=0 \\
u(x, 0)=\sin (x), \quad v(x, 0)=\cos (x)
\end{array}\right.
$$

where $x \in R, t \geq 0, \beta \in(0,1]$.

For $\beta=1$, the exact solutions of (37) are $u(x, t)=$ $\sin (x) e^{-t}$ and $v(x, t)=\cos (x) e^{-t}$.

As stated previously, we employ firstly the Laplace transform to both sides of (37), we can get the following Laplace system:

$$
\begin{aligned}
& U(x, s) \\
& =\frac{\sin (x)}{s}-\frac{1}{s^{\beta}} D_{x}^{2} U(x, s) \\
& +\frac{1}{s^{\beta}} \mathcal{L}\left\{\left(\mathcal{L}^{-1} U(x, s)\right)\left(\mathcal{L}^{-1} D_{x} U(x, s)\right)\right\} \\
& -\frac{1}{s^{\beta}} \mathcal{L}\left\{\left(\mathcal{L}^{-1} U(x, s)\right)\left(\mathcal{L}^{-1} V(x, s)\right)\right\}, \\
& V(x, s) \\
& =\frac{\cos (x)}{s}-\frac{1}{s^{\beta}} D_{x}^{2} V(x, s) \\
& +\frac{1}{s^{\beta}} \mathcal{L}\left\{\left(\mathcal{L}^{-1} V(x, s)\right)\left(\mathcal{L}^{-1} D_{x} V(x, s)\right)\right\} \\
& +\frac{1}{s^{\beta}} \mathcal{L}\left\{\left(\mathcal{L}^{-1} V(x, s)\right)\left(\mathcal{L}^{-1} V(x, s)\right)\right\} .
\end{aligned}
$$

Suppose that the $k$-th Laplace series solutions of (38) have the forms:

$$
\begin{array}{ll}
U_{k}(x, s)=\frac{\sin (x)}{s}+\sum_{m=1}^{k} \frac{u_{m}(x)}{s^{m \beta+1}}, \quad s>0, \\
V_{k}(x, s)=\frac{\cos (x)}{s}+\sum_{m=1}^{k} \frac{v_{m}(x)}{s^{m \beta+1}}, \quad s>0 .
\end{array}
$$

Consequently, the $k$-th Laplace fractional residual functions can be reformulated as:

$$
\begin{aligned}
\mathcal{L}\left\{\operatorname{Res}_{k}(x, s)\right\} & =\sum_{m=1}^{k} \frac{u_{m}(x)}{s^{m \beta+1}} \\
& -\frac{1}{s^{\beta}} D_{x}^{2}\left(\frac{\sin (x)}{s}+\sum_{m=1}^{k} \frac{u_{m}(x)}{s^{m \beta+1}}\right)
\end{aligned}
$$

$$
\begin{aligned}
& +\frac{1}{s^{\beta}} \mathcal{L}\left\{\left(\mathcal { L } ^ { - 1 } \left(\frac{\sin (x)}{s}\right.\right.\right. \\
& \left.\left.+\sum_{m=1}^{k} \frac{u_{m}(x)}{s^{m \beta+1}}\right)\right)\left(\mathcal { L } ^ { - 1 } D _ { x } \left(\frac{\sin (x)}{s}\right.\right. \\
& \left.\left.\left.+\sum_{m=1}^{k} \frac{u_{m}(x)}{s^{m \beta+1}}\right)\right)\right\} \\
& -\frac{1}{s^{\beta}} \mathcal{L}\left\{\left(\mathcal { L } ^ { - 1 } \left(\frac{\sin (x)}{s}\right.\right.\right. \\
& \left.\left.+\sum_{m=1}^{k} \frac{u_{m}(x)}{s^{m \beta+1}}\right)\right)\left(\mathcal { L } ^ { - 1 } \left(\frac{\cos (x)}{s}\right.\right. \\
& \left.\left.\left.+\sum_{m=1}^{k} \frac{v_{m}(x)}{s^{m \beta+1}}\right)\right)\right\} \\
& \mathcal{L}\left\{\operatorname{Res}_{k}(x, s)\right\}= \\
& =\sum_{m=1}^{k} \frac{v_{m}(x)}{s^{m \beta+1}} \\
& -\frac{1}{s^{\beta}} D_{x}^{2}\left(\frac{\cos (x)}{s}+\sum_{m=1}^{k} \frac{v_{m}(x)}{s^{m \beta+1}}\right) \\
& +\frac{1}{s^{\beta}} \mathcal{L}\left\{\left(\mathcal { L } ^ { - 1 } \left(\frac{\cos (x)}{s}\right.\right.\right. \\
& \left.\left.+\sum_{m=1}^{k} \frac{v(x)}{s^{m \beta+1}}\right)\right)\left(\mathcal { L } ^ { - 1 } D _ { x } \left(\frac{\cos (x)}{s}\right.\right. \\
& \left.\left.\left.+\sum_{m=1}^{k} \frac{v_{m}(x)}{s^{m \beta+1}}\right)\right)\right\} \\
& +\frac{1}{s^{\beta}} \mathcal{L}\left\{\left(\mathcal { L } ^ { - 1 } \left(\frac{\cos (x)}{s}\right.\right.\right. \\
& \left.\left.+\sum_{m=1}^{k} \frac{v_{m}(x)}{s^{m \beta+1}}\right)\right)\left(\mathcal { L } ^ { - 1 } \left(\frac{\sin (x)}{s}\right.\right. \\
& \left.\left.\left.+\sum_{m=1}^{k} \frac{u_{m}(x)}{s^{m \beta+1}}\right)\right)\right\} \text {. }
\end{aligned}
$$

For $k=1$, the $1^{\text {st }}$ - Laplace fractional residual 
functions will be as:

$$
\begin{aligned}
& \mathcal{L}\left\{\operatorname{Res}_{1}(x, s)\right\}=\frac{u_{1}(x)}{s^{\beta+1}} \\
& -\frac{1}{s^{\beta}}\left(\frac{\sin (x)}{s}+\frac{u_{1}^{\prime \prime}(x)}{s^{\beta+1}}\right) \\
& +\frac{1}{s^{\beta}} \mathcal{L}\left\{\left(\mathcal { L } ^ { - 1 } \left(\frac{\sin (x)}{s}\right.\right.\right. \\
& \left.\left.+\frac{u_{1}(x)}{s^{\beta+1}}\right)\right)\left(\mathcal { L } ^ { - 1 } \left(\frac{\sin (x)}{s}\right.\right. \\
& \left.\left.\left.+\frac{u_{1}^{\prime}(x)}{s^{\beta+1}}\right)\right)\right\} \\
& -\frac{1}{s^{\beta}} \mathcal{L}\left\{\left(\mathcal { L } ^ { - 1 } \left(\frac{\sin (x)}{s}\right.\right.\right. \\
& \left.\left.+\frac{u_{1}(x)}{s^{\beta+1}}\right)\right)\left(\mathcal { L } ^ { - 1 } \left(\frac{\cos (x)}{s}\right.\right. \\
& \left.\left.\left.+\frac{v_{1}(x)}{s^{\beta+1}}\right)\right)\right\} \text {, } \\
& \mathcal{L}\left\{\operatorname{Res}_{1}(x, s)\right\}=\frac{v_{1}(x)}{s^{\beta+1}} \\
& -\frac{1}{s^{\beta}} D_{x}^{2}\left(\frac{\cos (x)}{s}+\frac{v_{1}^{\prime \prime}(x)}{s^{\beta+1}}\right) \\
& +\frac{2}{s^{\beta}} \mathcal{L}\left\{\left(\mathcal { L } ^ { - 1 } \left(\frac{\cos (x)}{s}\right.\right.\right. \\
& \left.\left.+\frac{v_{1}(x)}{s^{\beta+1}}\right)\right)\left(\mathcal { L } ^ { - 1 } \left(\frac{\cos (x)}{s}\right.\right. \\
& \left.\left.\left.+\frac{v_{1}^{\prime}(x)}{s^{\beta+1}}\right)\right)\right\} \\
& +\frac{1}{s^{\beta}} D_{x} \mathcal{L}\left\{\left(\mathcal { L } ^ { - 1 } \left(\frac{\cos (x)}{s}\right.\right.\right. \\
& \left.\left.+\frac{v_{1}(x)}{s^{\beta+1}}\right)\right)\left(\mathcal { L } ^ { - 1 } \left(\frac{\sin (x)}{s}\right.\right. \\
& \left.\left.\left.+\frac{u_{1}(x)}{s^{\beta+1}}\right)\right)\right\} \text {. }
\end{aligned}
$$

Multiplication the obtained equations by $s^{\beta+1}$, we get

$$
\begin{aligned}
& s^{\beta+1} \mathcal{L}\left\{\operatorname{Res}_{1}(x, s)\right\} \\
&=\sin (x)+u_{1}(x)-\sin (x) \frac{v_{1}(x)}{s^{\beta}} \\
&+\sin (x) \frac{u_{1}^{\prime}(x)}{s^{\beta}} \\
&-\frac{\Gamma(2 \beta+1) u_{1}(x) v_{1}(x)}{\Gamma^{2}(\beta+1) s^{2 \beta}} \\
&+\frac{\Gamma(2 \beta+1) u_{1}(x) u_{1}^{\prime}(x)}{s^{\beta} \Gamma^{2}(\beta+1)}-\frac{u_{1}^{\prime \prime}(x)}{s^{\beta}}
\end{aligned}
$$

$$
\begin{aligned}
s^{\beta+1} \mathcal{L}\left\{\operatorname{Res}_{1}(x, s)\right\}= & \\
& =\cos (x)+v_{1}(x) \\
& +\cos (x) \frac{u_{1}(x)}{s^{\beta}} \\
& +\cos (x) \frac{v_{1}^{\prime}(x)}{s^{\beta}} \\
& -\frac{\Gamma(2 \beta+1) u_{1}(x) v_{1}(x)}{\Gamma^{2}(\beta+1) s^{2 \beta}} \\
& +\frac{\Gamma(2 \beta+1) v_{1}(x) v_{1}^{\prime}(x)}{s^{\beta} \Gamma^{2}(\beta+1)} \\
& -\frac{v_{1}^{\prime \prime}(x)}{s^{\beta}} .
\end{aligned}
$$

and by solving the systems

$\lim _{s \rightarrow \infty} s^{\beta+1} \mathcal{L}\left\{\operatorname{Res} U_{1}(x, s)\right\}=0$, and

$\lim _{s \rightarrow \infty} s^{\beta+1} \mathcal{L}\left\{\operatorname{Res} V_{1}(x, s)\right\}=0$, gives $u_{1}(x)=$

$-\sin (x)$, and $v_{1}(x)=-\cos (x)$.

For $k=2$, we have

$$
\begin{aligned}
s^{2 \beta+1} \mathcal{L}\left\{\operatorname{Res}_{2}(\right. & x, s)\} \\
& =-\sin (x)+u_{2}(x) \\
& -\sin (x) \frac{v_{2}(x)}{s^{\beta}} \\
& +\sin (x) \frac{u_{2}^{\prime}(x)}{s^{\beta}} \\
& -\frac{\Gamma(3 \beta+1) \sin (x) v_{2}(x)}{\Gamma(\beta+1) \Gamma(2 \beta+1) s^{2 \beta}} \\
& -\frac{\Gamma(4 \beta+1) u_{2}(x) v_{2}(x)}{s^{3 \beta} \Gamma^{2}(2 \beta+1)} \\
& -\frac{\Gamma(3 \beta+1) \sin (x) u_{2}^{\prime}(x)}{\Gamma(\beta+1) \Gamma(2 \beta+1) s^{2 \beta}} \\
& +\frac{\Gamma(4 \beta+1) u_{2}(x) u_{2}^{\prime}(x)}{s^{3 \beta} \Gamma^{2}(2 \beta+1)} \\
& -\frac{u_{2}^{\prime \prime}(x)}{s^{\beta}},
\end{aligned}
$$




$$
\begin{aligned}
s^{2 \beta+1} \mathcal{L}\left\{\operatorname{Res}_{2}(\right. & x, s)\} \\
& =-\cos (x)+v_{2}(x) \\
& +\cos (x) \frac{u_{2}(x)}{s^{\beta}} \\
& +\cos (x) \frac{v_{2}^{\prime}(x)}{s^{\beta}} \\
& -\frac{\Gamma(3 \beta+1) \cos (x) u_{2}(x)}{\Gamma(\beta+1) \Gamma(2 \beta+1) s^{2 \beta}} \\
& +\frac{\Gamma(4 \beta+1) u_{2}(x) v_{2}(x)}{s^{3 \beta} \Gamma^{2}(2 \beta+1)} \\
& -\frac{\Gamma(3 \beta+1) \cos (x) v_{2}^{\prime}(x)}{\Gamma(\beta+1) \Gamma(2 \beta+1) s^{2 \beta}} \\
& +\frac{\Gamma(4 \beta+1) v_{2}(x) v_{2}^{\prime}(x)}{s^{3 \beta} \Gamma^{2}(2 \beta+1)} \\
& -\frac{v_{2}^{\prime \prime}(x)}{s^{\beta}} .
\end{aligned}
$$

Depending on the fact $\lim _{s \rightarrow \infty} s^{2 \beta+1} \mathcal{L}\left\{\operatorname{Res} U_{2}(x, s)\right\}=$ 0 , and $\lim _{s \rightarrow \infty} s^{2 \beta+1} \mathcal{L}\left\{\operatorname{Res}_{2}(x, s)\right\}=0$, we get $u_{2}(x)=\sin (x)$, and $v_{2}(x)=\cos (x)$.

Following the procedure of Laplace RPS method, and by solving the system,

$\lim _{s \rightarrow \infty} s^{k \beta+1} \mathcal{L}\left\{\operatorname{Res}_{k}(x, s)\right\}=0$, and

$\lim _{s \rightarrow \infty} s^{k \beta+1} \mathcal{L}\left\{\operatorname{Res}_{k}(x, s)\right\}=0$, for the required

coefficients. On can conclude that $u_{k}(x)=$ $(-1)^{k} \sin (x)$, and $v_{k}(x)=(-1)^{k} \cos (x)$, for $k=$ $0,1,2, \ldots$. Therefore, the Laplace series solutions of (38) can be written as:

$$
\begin{array}{r}
\begin{aligned}
U(x, s)=\frac{\sin (x)}{s} & -\frac{\sin (x)}{s^{\beta+1}}+\frac{\sin (x)}{s^{2 \beta+1}} \\
& -\frac{\sin (x)}{s^{3 \beta+1}}+\cdots \\
& =\sin (x) \sum_{m=0}^{\infty} \frac{(-1)^{m}}{s^{m \beta+1}}, \\
V(x, s)=\frac{\cos (x)}{s} & -\frac{\cos (x)}{s^{\beta+1}}+\frac{\cos (x)}{s^{2 \beta+1}} \\
& -\frac{\cos (x)}{s^{3 \beta+1}}+\cdots \\
& =\cos (x) \sum_{m=0}^{\infty} \frac{(-1)^{m}}{s^{m \beta+1}},
\end{aligned}
\end{array}
$$

Table 3. Numerical results at $\beta=1$ and $n=10$ with different values of $t$ for Example 4.3.

\begin{tabular}{ccccc}
\hline $\boldsymbol{x}_{\boldsymbol{i}}$ & $\boldsymbol{t}_{\boldsymbol{i}}$ & $\boldsymbol{u}(\boldsymbol{x}, \boldsymbol{t})$ & $\boldsymbol{u}_{\mathbf{1 0}}(\boldsymbol{x}, \boldsymbol{t})$ & $\left|\boldsymbol{u}(\boldsymbol{x}, \boldsymbol{t})-\boldsymbol{u}_{\mathbf{1 0}}(\boldsymbol{x}, \boldsymbol{t})\right|$ \\
\hline \multirow{4}{*}{$\pi$} & 0.00 & 0.8660254037844386 & 0.8660254037844386 & 0.0 \\
$\overline{3}$ & 0.15 & 0.7453949728239977 & 0.7453949728239977 & 0.0 \\
& 0.30 & 0.6415673986967540 & 0.6415673986967916 & $3.752553823233029 \times 10^{-14}$ \\
& 0.45 & 0.5522021774725715 & 0.5522021774757754 & $3.203881604463276 \times 10^{-12}$ \\
& 0.60 & 0.4752848187499277 & 0.47528481882487794 & $7.495026821402462 \times 10^{-11}$
\end{tabular}

Finally, we operate the inverse Laplace transform to the Laplace series solutions (44), to conclude that the multiple fractional PS approximate solutions for the IVPs system (37) can be expressed as:

$$
\begin{aligned}
u(x, t)=\sin (x) & \left(1-\frac{t^{\beta}}{\Gamma(\beta+1)}\right. \\
+ & \frac{t^{2 \beta}}{\Gamma(2 \beta+1)}-\frac{t^{3 \beta}}{\Gamma(3 \beta+1)} \\
& \left.+\frac{t^{4 \beta}}{\Gamma(4 \beta+1)}+\cdots\right), \\
v(x, t)=\cos (x)\left(1-\frac{t^{\beta}}{\Gamma(\beta+1)}\right. & \left(\frac{t^{2 \beta}}{\Gamma(2 \beta+1)}-\frac{t^{3 \beta}}{\Gamma(3 \beta+1)}\right. \\
+ & \left.\frac{t^{4 \beta}}{\Gamma(4 \beta+1)}+\cdots\right),
\end{aligned}
$$

If we substitute $\beta=1$, then the multiple fractional PS solutions (45) reduce to

$$
\begin{gathered}
u(x, t)=\sin (x)\left(1-t+\frac{t^{2}}{2 !}-\frac{t^{3}}{3 !}+\frac{t^{4}}{4 !}\right. \\
+\cdots) . \\
v(x, t)=\cos (x)\left(1-t+\frac{t^{2}}{2 !}-\frac{t^{3}}{3 !}+\frac{t^{4}}{4 !}\right. \\
+\cdots) .
\end{gathered}
$$

which agree with Maclaurin series expansions of the exact solutions $u(x, t)=\sin (x) e^{-t}$, and $v(x, t)=$ $\cos (x) e^{-t}$. Further, the results are the same obtained in [36].

In Table 3, the numerical simulation of exact and tenth approximate solutions for IVP's system (37) is presented for various values of $t_{i}$ with step size 0.15 on $[0,1]$, fixed values of $x$, and for $\beta=1$. From the gained results, one can be noted that fully harmony between the obtained solutions by LRPS method and exact solutions. 


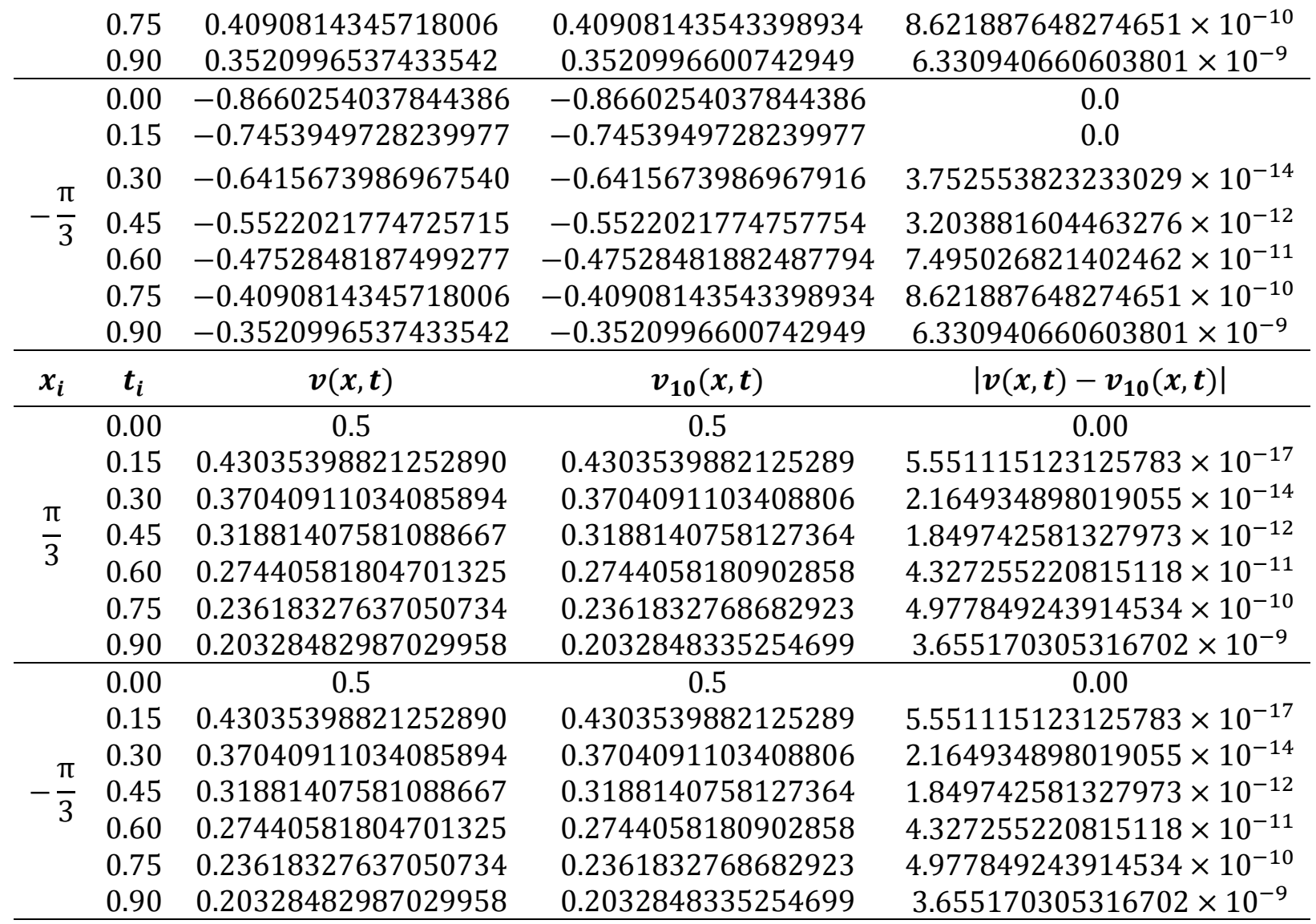

Furthermore, the curves of exact and multiple fractional PS approximate solutions for the IVPs system (37) for $\beta \in\{0.3,0.5,0.7,0.9,1\}$ have been drawn as shown Fig.3, when $t=1$. By increasing the $\beta$ values to the classical-order value $\beta=1$, we can note that the approximate solutions curves are consistent with each other and approach the exact curve.

(a)

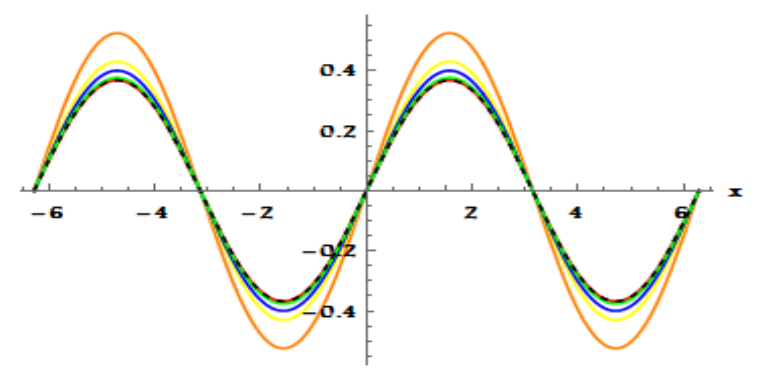

(b)

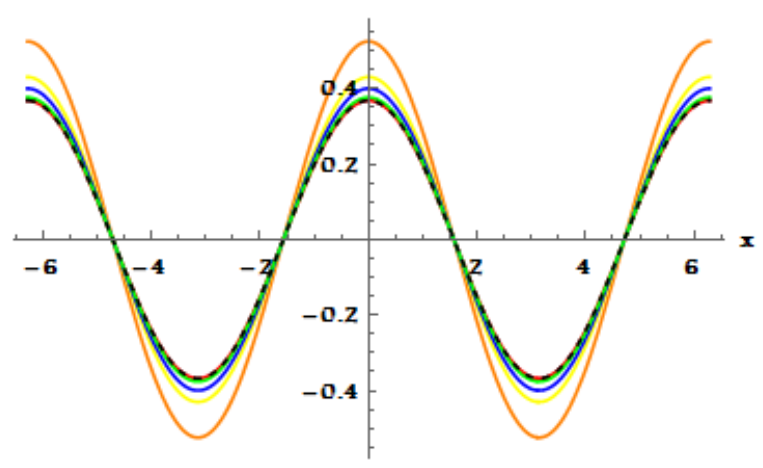

E-ISSN: 2224-2880

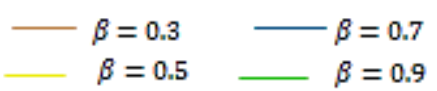

Fig. 3 (a) Curves of exact $u(x, t)$, and $10^{\text {th }}$ approximate solution $u_{10}(x, t)$, at various values of $\beta$ and $t=1$; (b) Curves of exact $v(x, t)$ and $10^{\text {th }}$ approximate solution $v_{10}(x, t)$ at various valuesof $\beta$ and $x=0$.

\section{Conclusion}

In this manuscript, the multiple fractional PS approximate solutions were profitably obtained for certain classes of FIVPs in terms of Caputo differentiability sense using the LRPS method. The effectiveness and applicability of the proposed algorithm have been investigated by doing linear and non-linear systems of FIVPs. The proposed algorithm produced accurate approximate solutions converge with the exact solutions without involving linearization, discretization, or any other limited conditions. There is an important point to make here regarding the practical applicability of the LRPS algorithm that it uses a new technicality easier and faster than the classical RPS algorithm in obtaining the coefficients of the proposed expansion, where 
one does not need to use the fractional derivatives through implementation some of mathematical operations in finding symbolic calculations and simulation of the problem via employing MATHEMATICA software. Obtained approximate solutions have been compatible with each other at various values of $\beta$, and the exact solutions. The results confirm that the LRPS algorithm is a straightforward and convenient tool to treat a various range of non-linear time fractional-PDEs that arise in engineering and science problems.

\section{References:}

[1] A. Kilbas, H. Srivastava and J. Trujillo, Theory and Applications of Fractional Differential Equations, Elsevier, Amsterdam, Netherlands, 2006.

[2] T. Abdeljawad, Fractional difference operators with discrete generalized Mittag-Leffler kernels, Chaos, Solitons and Fractals, Vol. 126, 2019, pp. 315-324.

[3] I. Podlubny, Fractional Differential Equations, Academic Press, San Diego, 1999.

[4] M. Al-Smadi, Simplified iterative reproducing kernel method for handling time-fractional BVPs with error estimation, Ain Shams Engineering Journal, Vol. 9, No. 4, 2018, pp. 2517-2525.

[5] O. Abu Arqub, and M. Al-Smadi, An adaptive numerical approach for the solutions of fractional advection-diffusion and dispersion equations in singular case under Riesz's derivative operator, Physica A: Statistical Mechanics and its Applications, Vol. 540, 2020, pp. 123257.

[6] S. Hasan, M. Al-Smadi, A. El-Ajou, S. Momani, S. Hadid and Z. Al-Zhour, Numerical approach in the Hilbert space to solve a fuzzy Atangana-Baleanu fractional hybrid system, Chaos, Solitons and Fractals, Vol. 143, 2021, pp. 110506.

[7] B. Bira, T.R. Sekhar and D. Zeidan, Exact solutions for some time- fractional evolution equations using Lie group theory, Mathematical Methods in the Applied Sciences, Vol. 41, No. 16, 2018 6717-pp. 6725.

[8] M. Al-Smadi, O. Abu Arqub and S. Hadid, An attractive analytical technique for coupled system of fractional partial differential equations in shallow water waves with conformable derivative, Communications in Theoretical Physics, Vol. 72, No. 8, 2020, pp. 085001.

[9] E. Abuteen, A. Freihat, M. Al-Smadi, H. Khalil and R. A. Khan, Approximate series solution of nonlinear, fractional Klein-Gordon equations using fractional reduced differential transform method, Journal of Mathematics and Statistics, Vol. 12, 2016, pp. 23-33.

[10] D. Zeidan, R. Touma and A. Slaouti, Application of Thermodynamically Compatible Two-Phase Flow
Model to the High-Resolution Simulations of Compressible Gas-Magma Flow, International Journal for Numerical Methods in Fluids, Vol. 76, No. 5, 2014, pp. 312-330.

[11] N. Laskin, Fractional quantum mechanics and Lévy path integrals, Physics Letters A, Vol. 268, No. 4-6, 2000, pp. 298-305.

[12] V. Parthiban and A. Manivannan, Analysis of Fractional Schrödinger Equation Occurring in Quantum Mechanics, Journal of Informatics and Mathematical Sciences, Vol. 9, 2017, pp. 595-607.

[13] M. Al-Smadi, O. Abu Arqub and S. Hadid, Approximate solutions of nonlinear fractional Kundu-Eckhaus and coupled fractional massive Thirring equations emerging in quantum field theory using conformable residual power series method, Physica Scripta, Vol. 95, No. 10, 2020, pp. 105205.

[14] I. Komashynska, M. Al-Smadi, O. Abu Arqub and S. Momani, An efficient analytical method for solving singular initial value problems of nonlinear systems, Applied Mathematics and Information Sciences, Vol. 10, No. 2, 2016, pp. 647-656.

[15] M. Al-Smadi, O. Abu Arqub and S. Momani, Numerical computations of coupled fractional resonant Schrödinger equations arising in quantum mechanics under conformable fractional derivative sense, Physica Scripta, Vol. 95, No. 7, 2020, pp. 075218.

[16] A. Freihet, S. Hasan, M. Al-Smadi, M. Gaith and S. Momani, Construction of fractional power series solutions to fractional stiff system using residual functions algorithm, Advances in Difference Equations, Vol. 2019, No. 1, 2019, pp. 1-15.

[17] N.A. Khan and T. Hameed, An Implementation of Haar Wavelet Based Method for Numerical Treatment of Time-fractional Schrodinger and Coupled Schrodinger Systems, IEEE/CAA Journal of Automatica Sinica 6, Vol. 2019, No. 1, 2019, pp. 177-187.

[18] S. Momani, O. Abu Arqub, A. Freihat, M. AlSmadi, Analytical approximations for FokkerPlanck equations of fractional order in multistep schemes, Applied and Computational Mathematics, Vol. 15, No. 3, 2016, pp. 319-330.

[19] M. Alaroud, M. Al-Smadi, R. R. Ahmad, U. K. Salma Din, An Analytical Numerical Method for Solving Fuzzy Fractional Volterra IntegroDifferential Equations, Symmetry, Vol. 11, No. 2, 2019, pp. 205.

[20] M. Alaroud, R. R. Ahmad, U. K. Salma Din, An efficient analytical-numerical technique for handling model of fuzzy differential equations of fractional-order, Filomat, Vol. 33, No. 2, 2019, pp. 617-632.

[21] M. S. Osman, A. Korkmaz, H. Rezazadeh, M. Mirzazadeh, M. Eslami, and Q. Zhou, The unified method for conformable time fractional Schrödinger equation with perturbation terms, Chinese Journal of Physics, Vol. 56. 2018, pp. 2500-2506. 
[22] A. Freihat, S. Hasan, M. Alaroud, M. Al-Smadi, R. R. Ahmad, U. K. Salma Din, Toward computational algorithm for time-fractional Fokker-Planck models, Advances in Mechanical Engineering, Vol. 11, No. 10, 2019, pp. 1-11.

[23] M. Alaroud, M. Al-smadi, R. R. Ahmad, U. K. Salma Din, Numerical computation of fractional Fredholm integro-differential equation of order $2 \beta$ arising in natural sciences, In Journal of Physics: Conference Series, Vol. 1212, No. 1, 2019, pp. 012022

[24] A. El-Ajou, Adapting the Laplace transform to create solitary solutions for the nonlinear timefractional dispersive PDEs via a new approach, The European Physical Journal Plus, Vol. 136, No. 2, (2021, pp. 1-22.

[25] T. Eriqat, A. El-Ajou, M. N. Oqielat, Z. Al-Zhour and S. Momani, A New Attractive Analytic Approach for Solutions of Linear and Nonlinear Neutral Fractional Pantograph Equations, Chaos, Solitons and Fractals, Vol. 138, 2020, pp. 109957.

[26] M. Alaroud, Application of Laplace residual power series method for approximate solutions of fractional IVP's, Alexandria Engineering Journal, 2021, DOI:10.1016/j.aej.2021.06.065.

[27] M. Alquran, M. Ali, M. Alsukhour and I. Jaradat, Promoted residual power series technique with Laplace transform to solve some time-fractional problems arising in physics, Results in Physics, Vol. 19, 2020, pp.103667.

[28] B. Tan and J. P. Boyd, Stability and long time evolution of the periodic solutions of the two coupled nonlinear Schrödinger equations. Chaos, Solitons, and Fractals Vol. 12, (2001), 721-734.

[29] M. Alaroud, M. Al-smadi, R. R. Ahmad, U. K. Salma Din, Computational optimization of residual power series algorithm for certain classes of fuzzy fractional differential equations, International Journal of Differential Equations, Vol. 2018, 2018, pp. 8686502.

[30] R. Saadeh, M. Alaroud, M. Al-smadi, R. R. Ahmad, U. K. Salma Din, Application of fractional residual power series algorithm to solve NewellWhitehead-Segel equation of fractional order, Symmetry, Vol. 11, No. 12, 2019, pp. 1431.

[31] M. Alaroud, O. Abu Arqub, R. Edwan, M. AlSmadi and S. Momani, Solving Fuzzy Fractional IVPs of order $2 \beta$ by Residual Power Series Algorithm. In 2019 IEEE Jordan International Joint Conference on Electrical Engineering and Information Technology (JEEIT), Vol. 2019, pp. 52-57.

[32] M. Al-Smadi, O. Abu Arqub, D. Zeidan, Fuzzy fractional differential equations under the MittagLeffler kernel differential operator of the ABC approach: Theorems and applications, Chaos, Solitons and Fractals, Vol. 146, 2021, pp. 110891.
[33] M. Al-Smadi, Fractional residual series for conformable time-fractional Sawada-Kotera-Ito, Lax, and Kaup-Kupershmidt equations of seventh order, Mathematical Methods in the Applied Sciences, 2021, DOI:10.1002/mma.7507

[34] A. Atangana and I. Koca, On the new fractional derivative and application to Nonlinear Baggs and Freedman model, Journal of Nonlinear Science and Applications, Vol. 9, 2016, pp. 2467-2480.

[35] A. Atangana, On the new fractional derivative and application to nonlinear Fisher's reaction-diffusion equation, Applied Mathematics and Computation, Vol. 273, 2016, pp. 948-956.

[36] D. B. Dhaigude, S. N. Kanade and C. D. Dhaigude, Solution for System Of Fractional Partial Differential Equations, Applications \& Applied Mathematics, Vol. 13, No. 1, 2018, pp. 21.

[37] A. Secer, M. A. Akinlar and A. Cevikel, Efficient solutions of systems of fractional PDEs by the differential transform method, Advances in Difference Equations, Vol. 2012, No. 1, 2012, pp. $1-7$.

\section{Contribution of individual authors to the creation of a scientific article (ghostwriting policy) \\ Mohammad Alaroud, carried out Conceptualization,} Investigation, Methodology, and Software.

Yousef Al-Qudah was responsible Writing-original draft, Writing-review, and editing.

\section{Sources of funding for research presented in a scientific article or scientific article itself}

This research was funded by Amman Arab University.

\section{Creative Commons Attribution License 4.0 (Attribution 4.0 International, CC BY 4.0)}

This article is published under the terms of the Creative Commons Attribution License 4.0 https://creativecommons.org/licenses/by/4.0/deed.en US 